\title{
Overview of the epidemiology and the threat of Klebsiella pneumoniae carbapenemases (KPC) resistance
}

\author{
Luke F Chen ${ }^{1-4}$ \\ Deverick J Anderson ${ }^{1-4}$ \\ David L Paterson ${ }^{5}$ \\ 'Duke Program for Infection \\ Prevention and Healthcare \\ Epidemiology, ${ }^{2}$ Duke Infection Control \\ Outreach Network, ${ }^{3}$ Duke University \\ Prevention Epicenter Program, \\ ${ }^{4}$ Division of Infectious Diseases and \\ International Health, Department of \\ Medicine, Duke University Medical \\ Center, Durham, NC, USA; ${ }^{5}$ University \\ of Queensland Centre for Clinical \\ Research (UQCCR), Royal Brisbane \\ and Women's Hospital Campus, \\ Brisbane, Australia
}

This article was published in the following Dove Press journal:

Infection and Drug Resistance

2I September 2012

Number of times this article has been viewed

\begin{abstract}
Klebsiella pneumoniae carbapenemases (KPCs) confer resistance to nearly all $\beta$-lactams. This broad-spectrum drug resistance mechanism has rapidly spread in the United States and is reportedly increasing elsewhere in the world. Thus, the emergence of KPC resistance is a major threat to global health. This article reviews the epidemiology and provides an overview of the dissemination of KPC-producing organisms.
\end{abstract}

Keywords: beta-lactam resistance, carbapenemase, drug resistance, epidemiology, treatment failure

\section{Introduction}

Carbapenems are often used to treat infections caused by Gram-negative bacteria that produce extended-spectrum $\beta$-lactamases (ESBL). A new class of bacterial enzymes capable of inactivating carbapenems, known as Klebsiella pneumoniae carbapenemases (KPCs), has rapidly spread in the United States and is increasing elsewhere in the world. KPCs are Ambler molecular class-A carbapenemases ${ }^{1}$ that typically reside on transferable plasmids and can hydrolyze all penicillins, cephalosporins, aztreonam, and carbapenems. Infections caused by KPCs have very limited options for treatment and often require the use of polymyxins, which fell into disuse in the 1970s due to high rates of nephrotoxicity. The epidemiology and molecular characteristics of KPC-producing organisms are still evolving and are intensively studied by researchers. The following review describes the current understanding of the epidemiology, genetic diversity, and clinical implications of the drug resistance mediated by KPC-type $\beta$-lactamases.

\section{Microbiologic and molecular characteristics of KPC-producing organisms}

Klebsiella pneumoniae is the most common organism associated with KPC resistance determinants. Molecular studies have shown that many K. pneumoniae isolates with KPC enzymes belong to a single clonal complex, $\mathrm{CC} 11$, and predominantly to a single sequence type, ST 258. ${ }^{2}$ However, KPCs are increasingly reported in other genera of the Enterobacteriaceae family, such as Escherichia, ${ }^{3}$ Proteus,${ }^{4}$ Serratia,${ }^{5}$ Salmonella,${ }^{6}$ and Citrobacter. ${ }^{7}$ Worse still, KPC resistance has been reported in inherently-resistant organisms such as Acinetobacter baumannii and Pseudomonas spp. ${ }^{8,9}$ For example, Akpaka and colleagues from Trinidad studied an isolate of multidrug-resistant P. aeruginosa that harbored a novel KPC-6 gene. ${ }^{10}$ Interestingly, the isolate was obtained from a patient who was hospitalized following a traumatic hip fracture who reported no
Correspondence: Luke F Chen

Duke University Medical Center,

Erwin Road, Durham, NC 277I0 USA

Tel + I 9| 96844596

Fax + 9196817494

Email luke.chen@duke.edu 
recent history of travel, suggesting that the specific isolate of KPC-producing Pseudomonas emerged locally, and likely continued to circulate in that region of the world.

The KPC gene was first identified from an isolate in North Carolina and was named KPC-1. ${ }^{11}$ Subsequent variants of the KPC gene were named in sequential numeric order from KPC-2 to KPC-11 (Table 1). However, a recent correction in the gene sequence of KPC-1 revealed that KPC-1 and KPC-2 were in fact identical enzymes; hence, the KPC-1 designation is no longer used. ${ }^{12}$

Evidence derived from minimal spanning tree analysis showed that KPC-2 was most likely the ancestral origin from which other KPC variants were derived. Currently identified KPC variants only differ from KPC-2 by minor nucleotide changes within four codons (nucleotides 147, 308, 716, and 814). ${ }^{13}$ For example, KPC-3 differs from KPC-2 by only one amino acid substitution (814C to $814 \mathrm{~T}$ ); KPC-6 differs from KPC-2 by a different amino acid substitution (716T to $716 \mathrm{G}) .^{14}$

KPC enzymes also differ in their kinetic properties and vary in their efficiency to hydrolyze different $\beta$-lactams. For example, KPC-2 appears to be a unique carbapenemase that is also not inhibited by currently available $\beta$-lactamase inhibitors such as clavulanic acid;15 KPC-3, however, demonstrates greatly heightened hydrolytic activity against ceftazidime (approximately 30 times higher than KPC-2 enzyme). ${ }^{16}$ Moreover, genetic changes associated with the $\mathrm{KPC}$ gene are stable in the wild and are increasingly common because antibiotic resistance conferred by KPC is adaptive for the organism against ongoing antibiotic selection pressure.

Of the 10 genetic variants of KPC identified thus far, KPC-2 and KPC-3 are the most common in clinical specimens and account for most epidemic outbreaks. KPC-2

Table I Klebsiella pneumoniae carbapenemase (KPC) resistance genotypes listed by year of first identification and geographic distribution

\begin{tabular}{lll}
\hline blaKPC & Year of identification & Distribution \\
\hline KPC-I & 1996 & USA (North Carolina) \\
KPC-2 & $1998-1999$ & $\begin{array}{l}\text { USA, Israel, China, Greece, Italy, } \\
\text { Brazil, France, Colombia, Taiwan } \\
\end{array}$ \\
KPC-3 & $2000-2001$ & USA, Israel \\
KPC-4 & 2003 & Puerto Rico, Scotland \\
KPC-5 & 2006 & Puerto Rico \\
KPC-6 & 2003 & Puerto Rico \\
KPC-7 & $2007-2008$ & USA \\
KPC-8 & 2008 & Puerto Rico \\
KPC-9 & 2009 & Israel \\
KPC-10 & 2009 & Puerto Rico \\
KPC-II & 2010 & Greece \\
\hline
\end{tabular}

appears to be more predominant worldwide, with outbreaks arising not only within the United States but also in Europe (especially Greece) and China. KPC-3 is mainly detected in the United States, Latin America, and Israel.

The rapid global dissemination of KPC genes is staggering. This observation can be attributed to a combination of three major social and microbiological mechanisms: international travel, patient-to-patient transmission of KPC-producing organisms, and interspecies transfer of KPC-resistant elements. ${ }^{17-19}$ Horizontal transfer of KPC resistance is a fascinating microbiological phenomenon that has devastating public health implications. Horizontal transfer of KPC can occur between bacteria because KPC resistance elements are often flanked by transposons (particularly Tn4401) and are carried on transferable plasmids of Gram-negative organisms. ${ }^{7,20}$ Worse still, many plasmids that carry KPC resistance elements concurrently carry other plasmidmediated resistance elements, such as quinolone (QnrA and QnrB) and aminoglycoside (rmtB) resistance. ${ }^{21,22}$

\section{Epidemiology}

Carbapenem resistance due to KPC has evolved rapidly since 2001. The distribution of KPC resistance determinants now vary substantially by geography. Although KPC is reported in most countries around the world, two divergent epidemiological patterns are seen: regions that report very few KPC-producing isolates (Australasia and Africa) and areas where KPCs are now considered endemic (northeastern USA, Puerto Rico, Greece, Zhejiang Province of China, and Israel).

\section{North America}

The first isolate of KPC-producing bacteria was discovered through the Intensive Care Antimicrobial Resistance Epidemiology (ICARE) surveillance program in a clinical specimen of K. pneumoniae from North Carolina in $1996 .{ }^{11}$ KPCs were infrequently isolated until 2001 when KPCproducing Enterobacteriaceae were reported in several extended clonal outbreaks in metropolitan hospitals located in the states of New York and New Jersey. ${ }^{23-25}$ Soon after, KPC-producing organisms spread rapidly along the east coast and then became widely disseminated westward and throughout the country. KPC-producing organisms have now been reported in 38 out of the 50 states of the US. ${ }^{26}$

Puerto Rico is a small island territory of the United States but it is surprisingly overrepresented with the number of reports and unique clones of KPC-producing organisms. For example, first isolates of KPC-4, -5, -6, -8, and -10 were 
identified in Puerto Rico. ${ }^{27}$ Furthermore, an island-wide surveillance study of $\beta$-lactam resistance in 2009 showed widespread KPC dissemination. Of 10,507 isolates submitted to the study, $12 \%$ of isolates were categorized as resistant to multiple beta-lactams. Notably, KPC was detected in 43\% of isolates with resistant to multiple beta-lactams, including isolates of Escherichia coli, K. pneumoniae, P. aeruginosa, and $A$. baumannii. ${ }^{28}$ KPC-producing organisms are truly endemic in Puerto Rico.

Canada, which shares a border with the United States, has only reported sporadic cases and limited outbreaks with KPC-producing organisms in hospitals in Ottawa, ${ }^{18}$ Toronto, ${ }^{29}$ and Montreal. ${ }^{30}$ Although the true epidemiology of KPC resistance in Canada is not completely understood, the prevalence of KPC resistance is thought to be low.

\section{Europe}

The first KPC-producing organism detected outside of the United States occurred in an isolate of $K$. pneumoniae that produced KPC-2 in Paris, France. ${ }^{17}$ Interestingly, the patient was hospitalized in a New York City hospital 2 months prior, suggesting that the resistant organism was imported from the USA. Subsequent surveillance data have shown that the prevalence of KPC organisms in Western Europe is generally low. However, there are several European regions with endemicity and outbreaks with KPC organisms. ${ }^{31}$

Greece has the highest prevalence of carbapenemresistant K. pneumoniae in Europe. In 2010, it was estimated that approximately $49 \%$ of all K. pneumoniae isolates from Greece reported to the European Centre for Disease Control and Prevention were carbapenem-resistant. KPC (particularly KPC-2)-producers were believed to be responsible for approximately $53 \%$ of the isolates with carbapenem resistance; VIM-producing organisms were responsible for the remaining 47\%. ${ }^{31-33}$ Epidemiological data from Athens, the capital of Greece, has reported disproportionately high numbers of KPC organisms. The city is thought to be an epicenter of carbapenem-resistant Enterobacteriaceae (CRE) in Greece.

The same 2010 European Centre for Disease Control and Prevention report showed that $15.2 \%$ of $K$. pneumoniae isolates in Italy were resistant to carbapenems. It is believed that Italy now has low-level KPC endemicity following the first report of KPC-3-producing $K$. pneumoniae in Tuscany in 2008. Indeed, dissemination of KPC producers continued relentlessly in Italy. In 2010, identical strains of KPCs were detected from seven different regions around Italy. ${ }^{34}$
The prevalence of carbapenem-resistant organisms in other parts of Europe is generally confined to sporadic imported cases. ${ }^{35}$ In the United Kingdom, the New Delhi metallo- $\beta$-lactamase 1 (NDM-1) seems to be the dominant carbapenemase among Enterobacteriaceae. ${ }^{34}$ In France, outbreaks involving organisms producing $\mathrm{KPC}-2$ enzymes have been reported; some of these French cases were imported from Greece or the USA. ${ }^{17,36,37}$

\section{Middle East}

The first report of KPC in Israel was in a returned traveler who received health care in New York in 2005. However, since the first reported case, both KPC-2 and KPC-3 have firmly established endemic status in Israel. ${ }^{38}$ Similarly to the USA, a single clone (ST258) of KPC-producing K. pneumoniae is responsible for most isolates in Israel. Fortunately, the Israeli government and its health care systems recognized the threat posed by KPCs and implemented a nationwide reporting system. Following the implementation of wideranging control measures, the occurrence of KPCs in Israel had reduced and stabilized. ${ }^{39}$

\section{South America}

The first report of KPC in South America was detected in a pair of KPC-2-producing $K$. pneumoniae isolates in Colombia in $2006 .{ }^{40}$ Since then, KPC-mediated resistance has become widespread in different parts of Colombia and has been reported in different clones of Enterobacteriaceae and Pseudomonas spp. ${ }^{41}$ Similarly, Brazil and Argentina have reported sporadic cases and clusters of infections with KPC-mediated resistance in different types of Gramnegative pathogens, including $K$. pneumoniae, E. coli, and Pseudomonas spp. ${ }^{42,43}$

\section{Asia}

As a region, Asia has widely disseminated KPC resistance elements. ${ }^{44}$ Both Korea and China have reported KPC in a variety of Gram-negative organisms, including Pseudomonas spp. and numerous genera of Enterobacteriaceae, including Proteus mirabilis, Enterobacter spp., Morganella morganii, Serratia marcescens, and Citrobacter freundii. ${ }^{45,46}$ Interestingly, the predominant type of $K$. pneumoniae associated with KPC production in China is ST11, which is closely related to ST258 found more commonly elsewhere in the world. ${ }^{47}$ Zhejiang, a southeastern province, appears to be the epicenter for KPC-producing organisms in China. ${ }^{22,48}$ Taiwan has reported imported cases of KPC related to visitors to the Zhejiang province in China. ${ }^{49}$ 
Although the Indian subcontinent has been linked as the source for imported cases of KPC to other countries (eg, France), recent data indicate carbapenem-resistance in this region is predominantly due to NDM- $1 .{ }^{50,51}$

\section{Australasia}

KPC-producing organisms have not been widely reported in Australia or New Zealand; sporadic cases have resulted from travel through KPC endemic areas. For example, the first isolate of KPC in Australia was detected in an isolate of $K$. pneumoniae from a returned traveler who was hospitalized during a holiday to Greece in the prior year. ${ }^{52}$ Similarly, the first isolate of KPC in New Zealand was $K$. pneumoniae harboring $\mathrm{KPC}-2$ resistance obtained from a patient repatriated from a Chinese hospital. ${ }^{53}$

\section{Clinical and epidemiologic characteristics of KPC infections - lessons from outbreaks}

KPC-producing organisms can colonize and cause infections similarly to their wild-type counterparts. Many infections are either systemic infections, occurring in patients with multiple invasive devices, or urinary tract infections without an indwelling catheter, particularly in immunocompromised patients. ${ }^{54,55}$

KPC-producing organisms rarely manifest as communityonset infections in nonendemic regions without any prior health care contact. ${ }^{56}$ Indeed, risk factors for colonization or infection with KPC-producing bacteria are all associated with health care, including exposure to antibiotic therapy, prolonged hospitalization, intensive care unit stay, immunosuppression, and organ transplantation. ${ }^{54,57,58}$

Data from an outbreak investigation in Chicago showed that admission to long-term care facilities was a risk factor for KPC transmission and acquisition. The investigators aptly summarized that long-term care facilities can act as a "point of convergence of patients at high risk; as an amplifier, by cross-transmission of KPC-positive cases; and as a facilitator of regional dissemination through discharge of patients harboring KPC-producing Enterobacteriaceae to multiple nursing homes", 3,59

\section{Detection of KPC-producing organisms}

The spread of KPC-producing organisms in health care settings represents a serious infection control issue. Detection of KPC-harboring organisms remains challenging due to several factors. First, KPC carbapenemases may not confer resistance to carbapenems but confer only reduced susceptibility. ${ }^{25,60}$ Additionally, various bacteria express KPC-encoded $\beta$-lactam resistance differently. For example, a KPC-producing $E$. coli isolate may have an ertapenem minimal inhibitory concentration of $1 \mathrm{mg} / \mathrm{L}$, whereas this value for a $\mathrm{KPC}$-producing $K$. pneumoniae may be $8 \mathrm{mg} / \mathrm{L}$. Thus, heterogeneous expression of KPCderived resistance among different bacteria makes it extremely challenging to designate a uniform cut-off point for phenotypic detection of resistance. These problems may be reduced with revision of carbapenem breakpoints for Enterobacteriaceae. ${ }^{61}$

Second, some phenotypic tests may mistakenly label KPC producers as ESBL producers. ${ }^{60}$ Third, imipenem- and meropenem-based automated susceptibility testing (AST) techniques may lack sensitivity for detecting KPC-producing organisms. A study from Israel showed that meropenembased susceptibility missed 24\% of KPC-producing Enterobacter spp. ${ }^{55}$ In contrast, cumulative data showed that ertapenem is the most suitable substrate for screening of carbapenemase activity, even though ertapenem resistance itself did not consistently correlate with KPC expression. Other causes of phenotypic ertapenem resistance may include coexistence of ESBLs or AmpC $\beta$-lactamases and outer membrane protein alterations. In light of increasing supportive evidence, laboratories using AST should switch to ertapenem to improve screening for KPC-producing Gram-negative bacilli. Laboratories must remain vigilant regarding the pitfalls of many AST systems whereby KPC carbapenemases may not be detected if low inocula of the organism are used in automated testing. ${ }^{25,54,62}$ Laboratories not using AST may consider a new CHROMagar (Paris, France) KPC screening technique that was shown to be $100 \%$ sensitive and $98.4 \%$ specific compared to KPC polymerase chain reaction (PCR). ${ }^{63}$

The final challenge in detecting KPC-producing organisms lies in the fact that isolates with positive results on screening tests still need to be confirmed as KPC producers. The modified Hodge test, a boronic acid-based test or a double-disc synergy test using $\beta$-lactamase inhibitors, such as clavulanic acid or tazobactam, have been used as confirmatory tests. ${ }^{64}$ These tests have limitations; paramount amongst these are that they are not specific for KPC production. Molecular tools, such as PCR, are the only way to definitively confirm the presence of genes encoding KPC resistance elements. 
These difficulties in KPC detection may lead to an underestimation of the true prevalence and incidence of KPC-producing organisms as well as the duration of a KPC epidemic.

\section{Implications for therapy and clinical outcomes of KPC infection}

KPC confers broad-spectrum resistance to $\beta$-lactams and carbapenems. Furthermore, KPC producers frequently carry additional genetic determinants, which confer resistance to other antibiotics, such as fluoroquinolones, aminoglycosides, and cotrimoxazole. . $21,33,48^{2}$

Few safe and practical therapeutic options remain for patients infected with KPC producers. Many clinicians have resorted to the use of tigecycline, polymyxins, and the few remaining aminoglycosides for the treatment of KPCproducing organisms. ${ }^{65}$

Tigecycline, a glycylcycline antibiotic, consistently shows in vitro activity against most isolates of KPC-producing organisms. However, several shortcomings limit the use of tigecycline. For example, tigecycline does not achieve high serum or urinary concentrations after infusion. ${ }^{66,67}$ A recent study placed additional uncertainty on the clinical efficacy of tigecycline as an antibiotic for hospital-acquired infections. Freire et al compared rates of curing hospital-acquired pneumonia in patients receiving tigecycline against those receiving imipenem-cilastatin. The multicenter, randomized, double-blind study showed that tigecycline was inferior to the comparator drug using a clinical evaluable study population. In addition, there were more gastrointestinal adverse effects (approximately twice as often as imipenem-cilastatin) ${ }^{68}$ Furthermore, a separate pooled analysis of all Phase III and IV studies of tigecycline showed higher all-cause mortality $(4.0 \%)$ in patients who received tigecycline compared to patients who received comparator drugs (3.0\%) $(95 \%$ confidence interval [CI]: 0.1-1.2). These findings have led to the issue of a black box warning from the US Food and Drug Administration. ${ }^{69}$ Finally, treatment emergent resistance to tigecycline has been reported; thus, clinicians should remain vigilant for clinically refractory infections when tigecycline is being used..$^{70}$ Despite these limitations, tigecycline has been used to successfully treat and cure patients infected with KPC-producers. ${ }^{71}$

Polymyxins, such as colistimethate and polymyxin B, are another class of drugs that have been used successfully to treat KPC-producers. These drugs are active against most genera of Gram-negative aerobic bacilli with the exception of Proteus spp. Additionally, polymyxins have poor activity against Serratia, Morganella, Providencia, Burkholderia, Vibrio, Brucella, Helicobacter, Moraxella, Aeromonas, and Edwardsiella.

Polymyxin B and colistin have been increasingly used, but they are associated with high rates of nephrotoxicity and have been considered drugs of last resort. There are recent data to show that the polymyxins may not be as nephrotoxic as previously thought; however, optimal dosing of polymyxins is not known. The optimal dosing regimens for colistin are currently under evaluation. ${ }^{72}$

Fosfomycin has been used successfully to treat KPCproducing organisms that still showed in vitro susceptibility. A study showed that fosfomycin retained in vitro activity against $93 \%$ of KPC-producing isolates collected in the USA in $2009 .{ }^{73}$ Notably, five out of six extremely drug-resistant KPC producers nonsusceptible to tigecycline and colistin were susceptible to fosfomycin. This drug causes very little toxicity and penetrates tissues readily; the concern with fosfomycin, however, is the propensity for resistance to rapidly develop when it is used in monotherapy. Thus, many investigators have proposed using fosfomycin in combination with other agents, such as aminoglycosides, since synergism has been demonstrated. ${ }^{74}$

Surveillance data show that the majority of KPCproducing isolates remain susceptible to polymyxins, and tigecycline. However, there are increasing worrisome reports of colistin-resistant and tigecycline-resistant isolates of KPC infections. For example, investigators in Greece reported that $18.6 \%$ of $301 \mathrm{KPC}$ isolates they studied were resistant to colistin and that the prevalence of colistin resistance among KPC producers had increased from $3.5 \%$ in 2008 to $20.8 \%$ in 2010. ${ }^{33}$ Furthermore, pan-resistant KPC-isolates have been sporadically reported around the world. ${ }^{75}$

Combination therapy of different antibiotics may improve the chances of cure in highly resistant infections. Separate analyses from researchers in Pittsburgh and from Greece demonstrated that combination therapy may be associated with improved survival and increased chance of cure of infection due to KPC-producers. ${ }^{76,77}$

Whether monotherapy or combination therapy is used, the outcomes associated with infections associated with KPC-producing organisms remain very poor compared to infections due to non-KPC-producing strains. For instance, the overall mortality in patients with KPCassociated infections has been estimated to be between $22 \%$ and $59 \%{ }^{78,79}$ 
A number of new antibiotics are under development for KPC producers. These include combinations of existing $\beta$-lactam antibiotics with new $\beta$-lactamase inhibitors able to inhibit KPC. These studies are now in Phase III clinical development. Additionally, neoglycosides (novel aminoglycosides) are under development and have activity against KPC producers.

\section{Prevention and infection control}

Containment of KPC-producing organisms is a key priority and has been a focus of intense study and policy making. The Centers for Disease Control and Prevention has updated its guidance for control of carbapenem-resistant Enterobacteriaceae (CRE). The interventions emphasize the need to develop region-wide activity and facility-level CRE prevention. ${ }^{64}$ The updated guidance recommends a set of core measures for all acute and long-term care facilities, including: (1) enhancing performance of hand hygiene, (2) placing CRE-colonized or CRE-infected patients on contact precautions, (3) judicious use of patient and staff cohorting if required, (4) minimize use of invasive medical devices, (5) promotion of antibiotic stewardship, and (6) screening for patients with risks for CRE. Facilities with documented transmission of CRE can undertake two additional measures: (1) active surveillance for CRE and (2) use of chlorhexidine bath or wipes.

A good example of success in controlling KPCs came from Kochar et $\mathrm{al}^{80}$ from the State University of New York Downstate Medical Center who used a combination of the above interventions to curtail an outbreak of multidrugresistant bacteria that included KPCs in their intensive care unit. The authors performed rectal swab screening for all new admissions to the intensive care unit and repeated the surveillance cultures weekly. KPC-infected or colonized patients were cohorted and were assigned dedicated nurses to care for them. Daily environmental cleaning was performed with a quaternary ammonium compound on all work surfaces in clinical areas. As a result of these interventions, the number of KPC infections/colonizations significantly decreased over the following 12 months.

\section{Conclusions}

Carbapenems have long been a reliable last line of defense in the treatment of infections caused by antimicrobialresistant Enterobacteriaceae. Thus, the emergence of KPC resistance is a major threat to global health. Recent results show that KPC genes are diverse, stable genetic elements, which can be difficult to detect. Furthermore, KPC-producing organisms can spread inside hospitals as well as outside in the community setting. Currently, few treatment options remain active against organisms that produce KPCs and have resulted in the increased use of tigecycline and polymyxins and intensified research on combination therapy. Until new effective drugs or combinations of drugs are found, detection, prevention, and containment are the keys to curtailing the spread of this dangerous antimicrobial resistance.

\section{Disclosure}

DA has received research grants from Merck and Pfizer, Inc, and has participated on the speaker's bureau for Merck. He receives royalties from UpToDate Online. He was supported by the Robert Wood Johnson Foundation Physician Faculty Scholar's Program and a K23 from the NIAID (1K23AI095357-01). LC has received a research grant from Merck, Inc, is a speaker for Cubist and Optimer Pharmaceuticals, a consultant and writes for Medscape, and receives royalties from UpToDate Online. DP has received research support for other projects and has appeared on advisory boards for Merck, AstraZeneca, Cubist, Pfizer, Novartis, and Leo Pharmaceuticals.

\section{References}

1. Ambler RP, Coulson AF, Frère JM, et al. A standard numbering scheme for the class A beta-lactamases. Biochem J. 1991;276(Pt 1):269-270.

2. Kitchel B, Rasheed JK, Patel JB, et al. Molecular epidemiology of KPC-producing Klebsiella pneumoniae isolates in the United States: clonal expansion of multilocus sequence type 258. Antimicrob Agents Chemother. 2009;53(8):3365-3370.

3. Urban C, Meyer KS, Mariano N, et al. Identification of TEM-26 betalactamase responsible for a major outbreak of ceftazidime-resistant Klebsiella pneumoniae. Antimicrob Agents Chemother. 1994;38(2): 392-395

4. Tibbetts R, Frye JG, Marschall J, Warren D, Dunne W. Detection of KPC-2 in a clinical isolate of proteus mirabilis and first reported description of carbapenemase resistance caused by a KPC beta-lactamase in P. mirabilis. J Clin Microbiol. 2008;46(9):3080-3083.

5. Zhang R, Zhou HW, Cai JC, Chen GX. Plasmid-mediated carbapenemhydrolysing beta-lactamase KPC-2 in carbapenem-resistant Serratia marcescens isolates from Hangzhou, China. $J$ Antimicrob Chemother. 2007;59(3):574-576

6. Miriagou V, Tzouvelekis LS, Rossiter S, Tzelepi E, Angulo FJ, Whichard JM. Imipenem resistance in a Salmonella clinical strain due to plasmid-mediated class A carbapenemase KPC-2. Antimicrob Agents Chemother. 2003;47(4):1297-1300.

7. Rasheed JK, Biddle JW, Anderson KF, et al. Detection of the Klebsiella pneumoniae carbapenemase type 2 carbapenem-hydrolyzing enzyme in clinical isolates of Citrobacter freundii and $\mathrm{K}$. oxytoca carrying a common plasmid. J Clin Microbiol. 2008;46(6):2066-2069.

8. Villegas MV, Lolans K, Correa A, Kattan JN, Lopez JA, Quinn JP. First identification of Pseudomonas aeruginosa isolates producing a KPC-type carbapenem-hydrolyzing beta-lactamase. Antimicrob Agents Chemother. 2007;51(4):1553-1555.

9. Sacha P, Ostas A, Jaworowska J, et al. The KPC type beta-lactamases: new enzymes that confer resistance to carbapenems in Gram-negative bacilli. Folia Histochem Cytobiol. 2009;47(4):537-543. 
10. Akpaka PE, Swanston WH, Ihemere HN, et al. Emergence of KPCproducing Pseudomonas aeruginosa in Trinidad and Tobago. J Clin Microbiol. 2009;47(8):2670-2671.

11. Yigit H, Queenan AM, Anderson GJ, et al. Novel carbapenemhydrolyzing beta-lactamase, KPC-1, from a carbapenem-resistant strain of Klebsiella pneumoniae. Antimicrob Agents Chemother. 2001;45(4): 1151-1161.

12. Yigit H, Queenan AM, Anderson GJ, et al. Author's correction- Novel carbapenem-hydrolyzing beta-lactamase, KPC-1, from a carbapenemresistant strain of Klebsiella pneumoniae. Antimicrob Agents Chemother. 2008;52(2).

13. Chen L, Mediavilla JR, Endimiani A, et al. Multiplex real-time PCR assay for detection and classification of Klebsiella pneumoniae carbapenemase gene (bla KPC) variants. J Clin Microbiol. 2011;49(2): 579-585.

14. Wolter DJ, Kurpiel PM, Woodford N, Palepou MF, Goering RV, Hanson ND. Phenotypic and enzymatic comparative analysis between the novel KPC variant, KPC-5, and its evolutionary variants, KPC-2 and KPC-4. Antimicrob Agents Chemother. 2009;53(2):557-562.

15. Papp-Wallace KM, Bethel CR, Distler AM, Kasuboski C, Taracila M, Bonomo RA. Inhibitor resistance in the KPC-2 beta-lactamase, a preeminent property of this class A beta-lactamase. Antimicrob Agents Chemother. 2010;54(2):890-897.

16. Alba J, Ishii Y, Thomson K, Moland ES, Yamaguchi K. Kinetics study of KPC-3, a plasmid-encoded class A carbapenem-hydrolyzing beta-lactamase. Antimicrob Agents Chemother. 2005;49(11): 4760-4762.

17. Naas T, Nordmann P, Vedel G, Poyart C. Plasmid-mediated carbapenemhydrolyzing beta-lactamase KPC in a Klebsiella pneumoniae isolate from France. Antimicrob Agents Chemother. 2005;49(10): 4423-4424.

18. Goldfarb D, Harvey SB, Jessamine K, Jessamine P, Toye B, Desjardins M. Detection of plasmid-mediated KPC-producing Klebsiella pneumoniae in Ottawa, Canada: evidence of intrahospital transmission. J Clin Microbiol. 2009;47(6):1920-1922.

19. Sidjabat HE, Silveira FP, Potoski BA, et al. Interspecies spread of Klebsiella pneumoniae carbapenemase gene in a single patient. Clin Infect Dis. 2009;49(11):1736-1738.

20. Naas T, Cuzon G, Villegas MV, Lartigue MF, Quinn JP, Nordmann P. Genetic structures at the origin of acquisition of the beta-lactamase bla KPC gene. Antimicrob Agents Chemother. 2008;52(4):1257-1263.

21. Endimiani A, Carias LL, Hujer AM, et al. Presence of plasmid-mediated quinolone resistance in Klebsiella pneumoniae isolates possessing blaKPC in the United States. Antimicrob Agents Chemother. 2008;52(7) 2680-2682.

22. Sheng JF, Li JJ, Tu S, et al. blaKPC and $\mathrm{rmtB}$ on a single plasmid in Enterobacter amnigenus and Klebsiella pneumoniae isolates from the same patient. Eur J Clin Microbiol Infect Dis. 2012;31(7):1585-1591.

23. Bratu S, Brooks S, Burney S, et al. Detection and spread of Escherichia coli possessing the plasmid-borne carbapenemase KPC-2 in Brooklyn, New York. Clin Infect Dis. 2007;44(7):972-975.

24. Lomaestro BM, Tobin EH, Shang W, Gootz T. The spread of Klebsiella pneumoniae carbapenemase-producing K. pneumoniae to upstate New York. Clin Infect Dis. 2006;43(3):e26-e28.

25. Bratu S, Mooty M, Nichani S, et al. Emergence of KPC-possessing Klebsiella pneumoniae in Brooklyn, New York: epidemiology and recommendations for detection. Antimicrob Agents Chemother. 2005; 49(7):3018-3020.

26. Centers for Disease Control and Prevention. Public Health update of Carbapenem-Resistant Enterobacteriaceae (CRE) producing metallobeta-lactamases (NDM, VIM, IMP) in the US reported to CDC 2011. Available from: http://www.cdc.gov/HAI/organisms/cre.html. Accessed on June 21, 2012.

27. Robledo IE, Vázquez GJ, Moland EE, et al. Dissemination and molecular epidemiology of KPC-producing Klebsiella pneumoniae collected in Puerto Rico Medical Center Hospitals during a 1-year period. Epidemiol Res Intl. 2011;1-8.
28. Robledo IE, Aquino EE, Vázquez GJ. Detection of the KPC gene in Escherichia coli, Klebsiella pneumoniae, Pseudomonas aeruginosa, and Acinetobacter baumannii during a PCR-based nosocomial surveillance study in Puerto Rico. Antimicrob Agents Chemother. 2011;55(6): 2968-2970.

29. Pillai DR, Melano R, Rawte P, et al. Klebsiella pneumoniae Carbapenemase, Canada. Emerg Infect Dis. 2009;15(5):827-829.

30. Tricky new superbug making inroads in Canada. 2011. Available from: http://m.ctv.ca/topstories/20110918/superbug-outrebak-canadaklebsiella-pneumoniae-1109018.html. Accessed on June 14, 2012.

31. European Centre for Disease Prevention and Control. Surveillance Report - Antimicrobial resistance surveillance in Europe, 2010. 2011. Available from: http:/www.ecdc.europa.eu/en/publications/Publications/1111_SUR_AMR_data.pdf.pdf. Accessed on June 16, 2012.

32. Mavroidi A, Neonakis I, Liakopoulos A, et al. Detection of Citrobacter koseri carrying beta-lactamase KPC-2 in a hospitalised patient, Greece, Jul 2011. Euro Surveill. 2011;16(41):pii:19990.

33. Zagorianou A, Sianou E, Iosifidis E, et al. Microbiological and molecular characteristics of carbapenemase-producing Klebsiella pneumoniae endemic in a tertiary Greek hospital during 2004-2010. Euro Surveill. 2012;17(7):pii:20088.

34. Canton R, Akova M, Carmeli Y, et al. Rapid evolution and spread of carbapenemases among Enterobacteriaceae in Europe. Clin Microbiol Infect. 2012;18(5):413-431.

35. Woodford N, Zhang J, Warner M, et al. Arrival of Klebsiella pneumoniae producing KPC carbapenemase in the United Kingdom. $J$ Antimicrob Chemother. 2008;62(6):1261-1264.

36. Carbonne A, Thiolet JM, Fournier S, et al. Control of a multi-hospital outbreak of KPC-producing Klebsiella pneumoniae type 2 in France, September to October 2009. Euro Surveill. 2010;15(48): pii:19734.

37. Kassis-Chikhani N, Decre D, Ichai P, et al. Outbreak of Klebsiella pneumoniae producing KPC-2 and SHV-12 in a French hospital. $J$ Antimicrob Chemother. 2010;65(7):1539-1540.

38. Leavitt A, Navon-Venezia S, Chmelnitsky I, Schwaber MJ, Carmeli Y. Emergence of KPC-2 and KPC-3 in carbapenem-resistant Klebsiella pneumoniae strains in an Israeli hospital. Antimicrob Agents Chemother. 2007;51(8):3026-3029.

39. Ciobotaro P, Oved M, Nadir E, Bardenstein R, Zimhony O. An effective intervention to limit the spread of an epidemic carbapenem-resistant Klebsiella pneumoniae strain in an acute care setting: from theory to practice. Am J Infect Control. 2011;39(8):671-677.

40. Villegas MV, Lolans K, Correa A, et al. First detection of the plasmidmediated class A carbapenemase KPC-2 in clinical isolates of Klebsiella pneumoniae from South America. Antimicrob Agents Chemother. 2006; 50(8):2880-2882.

41. Cuzon G, Naas T, Villegas MV, Correa A, Quinn JP, Nordmann P. Wide dissemination of Pseudomonas aeruginosa producing beta-lactamase blaKPC-2 gene in Colombia. Antimicrob Agents Chemother. 2011; 55(11):5350-5353.

42. Peirano G, Seki LM, Val Passos VL, Pinto MC, Guerra LR, Asensi MD. Carbapenem-hydrolysing beta-lactamase KPC-2 in Klebsiella pneumoniae isolated in Rio de Janeiro, Brazil. JAntimicrob Chemother. 2009;63(2):265-268.

43. Pasteran F, Faccone D, Gomez S, et al. Detection of an international multiresistant clone belonging to sequence type 654 involved in the dissemination of KPC-producing Pseudomonas aeruginosa in Argentina. J Antimicrob Chemother. 2012;67(5):1291-1293.

44. Jean SS, Hsueh PR. High burden of antimicrobial resistance in Asia. Int J Antimicrob Agents. 2011;37(4):291-295.

45. Cai JC, Zhou HW, Zhang R, Chen GX. Emergence of Serratia marcescens, Klebsiella pneumoniae, and Escherichia coli Isolates possessing the plasmid-mediated carbapenem-hydrolyzing beta-lactamase KPC-2 in intensive care units of a Chinese hospital. Antimicrob Agents Chemother. 2008;52(6):2014-2018.

46. Lee HK, Park YJ, Kim JY, et al. Prevalence of decreased susceptibility to carbapenems among Serratia marcescens, Enterobacter cloacae, and Citrobacter freundii and investigation of carbapenemases. Diagn Microbiol Infect Dis. 2005;52(4):331-336. 
47. QiY, Wei Z, Ji S, Du X, Shen P, YuY. ST11, the dominant clone of KPCproducing Klebsiella pneumoniae in China. J Antimicrob Chemother. 2011;66(2):307-312.

48. Hu YY, Cai JC, Zhang R, Zhou HW, Sun Q, Chen GX. Emergence of Proteus mirabilis harboring blaKPC-2 and qnrD in a Chinese Hospital. Antimicrob Agents Chemother. 2012;56(5):2278-2282.

49. Chung KP, Tseng SP, Huang YT, Tsai TH, Teng LJ, Hsueh PR. Arrival of Klebsiella pneumoniae carbapenemase (KPC)-2 in Taiwan. J Antimicrob Chemother. 2011;66(5):1182-1184.

50. Potron A, Poirel L, Verdavaine D, Nordmann P. Importation of KPC-2producing Escherichia coli from India. J Antimicrob Chemother. 2012; 67(1):242-243.

51. Sidjabat H, Nimmo GR, Walsh TR, et al. Carbapenem resistance in Klebsiella pneumoniae due to the New Delhi Metallo-beta-lactamase. Clin Infect Dis. 2011;52(4):481-484.

52. Huntington P, Coatsworth N, Hardiman R, Hudson B, Kotsiou G, Fernandes C. Klebsiella pneumoniae carbapenemase in Australia: detection of a KPC-producing clinical isolate at a Sydney hospital. The Australian Society for Microbiology (ASM) 2011 Annual Conference. Hobart, Tasmania, Australia; 2011.

53. Institute of Environmental Science and Research. New Zealand Public Health Surveillance Report. 2011. Available from: http://www.surv. esr.cri.nz/PDF_surveillance/NZPHSR/2011/NZPHSR2011March.pdf. Accessed March 12, 2011.

54. Bratu S, Landman D, Haag R, et al. Rapid spread of carbapenem-resistant Klebsiella pneumoniae in New York City: a new threat to our antibiotic armamentarium. Arch Intern Med. 2005;165(12):1430-1435.

55. Marchaim D, Navon-Venezia S, Schwaber MJ, Carmeli Y. Isolation of imipenem-resistant Enterobacter species: emergence of KPC-2 carbapenemase, molecular characterization, epidemiology, and outcomes. Antimicrob Agents Chemother. 2008;52(4):1413-1418.

56. Tsakris A, Poulou A, Markou F, et al. Dissemination of clinical isolates of Klebsiella oxytoca harboring CMY-31, VIM-1, and a New OXY-2-type variant in the community. Antimicrob Agents Chemother. 2011;55(7):3164-3168.

57. Woodford N, Tierno PM Jr, Young K, et al. Outbreak of Klebsiella pneumoniae producing a new carbapenem-hydrolyzing class A betalactamase, KPC-3, in a New York Medical Center. Antimicrob Agents Chemother. 2004;48(12):4793-4799.

58. Chia JH, Su LH, Lee MH, et al. Development of high-level carbapenem resistance in Klebsiella pneumoniae among patients with prolonged hospitalization and carbapenem exposure. Microb Drug Resist. 2010; 16(4):317-325.

59. Won SY, Munoz-Price LS, Lolans K, Hota B, Weinstein RA, Hayden MK. Emergence and rapid regional spread of Klebsiella pneumoniae carbapenemase-producing Enterobacteriaceae. Clin Infect Dis. 2011; 53(6):532-540.

60. Smith Moland E, Hanson ND, Herrera VL, et al. Plasmid-mediated, carbapenem-hydrolysing beta-lactamase, KPC-2, in Klebsiella pneumoniae isolates. J Antimicrob Chemother. 2003;51(3): 711-714.

61. Institute. CaLS. Performance Standards for Antimicrobial Susceptibility Testing: Twenty-Second Informational Supplement. 2012. Available from: http://www.clsi.org/source/orders/free/m100-s22.pdf. Accessed July 29, 2012.

62. Tenover FC, Kalsi RK, Williams PP, et al. Carbapenem resistance in Klebsiella pneumoniae not detected by automated susceptibility testing. Emerg Infect Dis. 2006;12(8):1209-1213.

63. Samra Z, Bahar J, Madar-Shapiro L, Aziz N, Israel S, Bishara J. Evaluation of CHROMagar KPC for rapid detection of carbapenem-resistant Enterobacteriaceae. J Clin Microbiol. 2008;46(9):3110-3111.

64. Centers for Disease Control and Prevention. 2012 CRE Toolkit-Guidance for Control of Carbapenem-resistant Enterobacteriaceae (CRE). 2012. Available from: http://www.cdc.gov/hai/organisms/cre/cre-toolkit/f-levelprevention-supmeasures.html. Accessed July 29, 2012.
65. Kelesidis T, Karageorgopoulos DE, Kelesidis I, Falagas ME. Tigecycline for the treatment of multidrug-resistant Enterobacteriaceae: a systematic review of the evidence from microbiological and clinical studies. $J$ Antimicrob Chemother. 2008;62(5):895-904.

66. Burkhardt O, Rauch K, Kaever V, Hadem J, Kielstein JT, Welte T. Tigecycline possibly underdosed for the treatment of pneumonia: a pharmacokinetic viewpoint. Int J Antimicrob Agents. 2009;34(1):101-102.

67. Peleg AY, Potoski BA, Rea R, et al. Acinetobacter baumannii bloodstream infection while receiving tigecycline: a cautionary report. $J$ antimicrob Chemother. 2007;59(1):128-131.

68. Freire AT, Melnyk V, Kim MJ, et al. Comparison of tigecycline with imipenem/cilastatin for the treatment of hospital-acquired pneumonia. Diagn Microbiol Infect Dis. 2010;68(2):140-151.

69. Food and Drug Administration. FDA Drug Safety Communication: Increased risk of death with Tygacil (tigecycline) compared to other antibiotics used to treat similar infections. 2010. Available from: http:// www.fda.gov/Drugs/DrugSafety/ucm224370.htm. Accessed July 29, 2012.

70. Spanu T, De Angelis G, Cipriani M, et al. In vivo emergence of tigecycline resistance in multidrug-resistant Klebsiella pneumoniae and Escherichia coli. Antimicrob Agents Chemother. 2012;56(8):4516-4518.

71. Tumbarello M, Viale P, Viscoli C, et al. Predictors of mortality in bloodstream infections caused by Klebsiella pneumoniae carbapenemaseproducing K. pneumoniae: importance of combination therapy. Clin Infect Dis. July 2, 2012. doi:10.1093/cid/cis588.

72. Garonzik SM, Li J, Thamlikitkul V, et al. Population pharmacokinetics of colistin methanesulfonate and formed colistin in critically ill patients from a multicenter study provide dosing suggestions for various categories of patients. Antimicrob Agents Chemother. 2011;55(7): 3284-3294.

73. Endimiani A, Patel G, Hujer KM, et al. In vitro activity of fosfomycin against blaKPC-containing Klebsiella pneumoniae isolates, including those nonsusceptible to tigecycline and/or colistin. Antimicrob Agents Chemother. 2010;54(1):526-529.

74. Cai Y, Fan Y, Wang R, An MM, Liang BB. Synergistic effects of aminoglycosides and fosfomycin on Pseudomonas aeruginosa in vitro and biofilm infections in a rat model. J Antimicrob Chemother. 2009; 64(3):563-566.

75. Humphries RM, Kelesidis T, Dien Bard J, Ward KW, Bhattacharya D, Lewinski MA. Successful treatment of pan-resistant Klebsiella pneumoniae pneumonia and bacteraemia with a combination of high-dose tigecycline and colistin. J Med Microbiol. 2010;59(Pt 11):1383-1386.

76. Qureshi ZA, Paterson DL, Potoski BA, et al. Treatment outcome of bacteremia due to KPC-producing Klebsiella pneumoniae: superiority of combination antimicrobial regimens. Antimicrob Agents Chemother. 2012;56(4):2108-2113.

77. Zarkotou O, Pournaras S, Tselioti P, et al. Predictors of mortality in patients with bloodstream infections caused by KPC-producing Klebsiella pneumoniae and impact of appropriate antimicrobial treatment. Clin Microbiol Infect. 2011;17(12):1798-1803.

78. Chen LF, Anderson DJ, Richmond BB, et al. Outbreak of Klebsiella pneumoniae carbapenemase producing organisms in a community hospital in Virginia. Paper presented at: 48th Annual ICAAC/IDSA 46th Annual Meeting: A Joint Meeting of the American Society for Microbiology and the Infectious Diseases Society of America, 2008; Washington, DC.

79. Lee J, Patel G, Ye F, et al. Is there a difference in clinical outcomes for KPC-2 versus KPC-3? Paper presented at: 48th Annual ICAAC/IDSA 46th Annual Meeting: A Joint Meeting of the American Society for Microbiology and the Infectious Diseases Society of America, 2008; Washington, DC.

80. Kochar S, Sheard T, Sharma R, et al. Success of an infection control program to reduce the spread of carbapenem-resistant Klebsiella pneumoniae. Infect Control Hosp Epidemiol. 2009;30(5):447-52. 
Infection and Drug Resistance

\section{Publish your work in this journal}

Infection and Drug Resistance is an international, peer-reviewed openaccess journal that focuses on the optimal treatment of infection (bacterial, fungal and viral) and the development and institution of preventive strategies to minimize the development and spread of resistance. The journal is specifically concerned with the epidemiology of antibiotic

resistance and the mechanisms of resistance development and diffusion in both hospitals and the community. The manuscript management system is completely online and includes a very quick and fair peerreview system, which is all easy to use. Visit http://www.dovepress.com/ testimonials.php to read real quotes from published authors.

Submit your manuscript here: http://www.dovepress.com/infection-and-drug-resistance-journal 